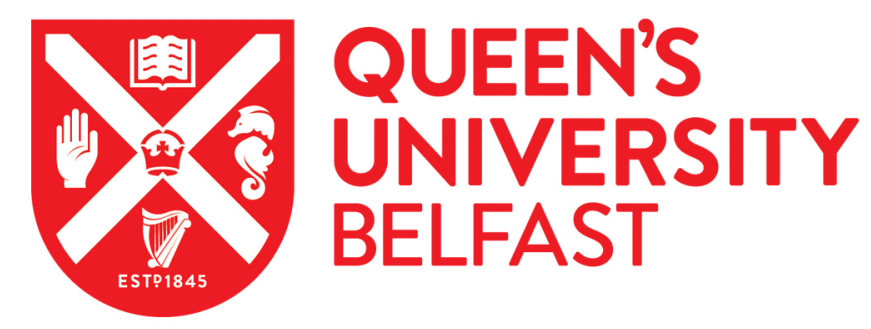

\title{
Mutations in TNFRSF11A, affecting the signal peptide of RANK, cause familial expansile osteolysis
}

Hughes, A., Ralston, S. H., Marken, J., Bell, C., McPherson, H., Wallace, R. G. H., Van Hul, W., Whyte, M. P., Nakatsuka, K., Hovy, L., \& Anderson, D. M. (2000). Mutations in TNFRSF11A, affecting the signal peptide of RANK, cause familial expansile osteolysis. Nature Genetics, 24(1), 45-48.

Published in:

Nature Genetics

Queen's University Belfast - Research Portal:

Link to publication record in Queen's University Belfast Research Portal

\section{General rights}

Copyright for the publications made accessible via the Queen's University Belfast Research Portal is retained by the author(s) and / or other copyright owners and it is a condition of accessing these publications that users recognise and abide by the legal requirements associated with these rights.

Take down policy

The Research Portal is Queen's institutional repository that provides access to Queen's research output. Every effort has been made to ensure that content in the Research Portal does not infringe any person's rights, or applicable UK laws. If you discover content in the Research Portal that you believe breaches copyright or violates any law, please contact openaccess@qub.ac.uk. 


\title{
Mutations in TNFRSF11A, affecting the signal peptide of RANK, cause familial expansile osteolysis
}

\author{
Anne E. Hughes ${ }^{1}$, Stuart H. Ralston ${ }^{2}$, John Marken ${ }^{3}$, Christine Bell ${ }^{2}$, Heather MacPherson ${ }^{2}$, \\ Richard G.H. Wallace ${ }^{4}$, Wim van Hul ${ }^{5}$, Michael P. Whyte ${ }^{6}$, Kyoshi Nakatsuka $^{7}$, Louis Hovy ${ }^{8}$ \& Dirk M. Anderson ${ }^{3}$
}

Familial expansile osteolysis 1,2 (FEO, MIM 174810) is a rare, autosomal dominant bone disorder characterized by focal areas of increased bone remodelling. The osteolytic lesions, which develop usually in the long bones during early adulthood, show increased osteoblast and osteoclast activity. Our previous linkage studies mapped the gene responsible for FEO to an interval of less than $5 \mathrm{cM}$ between D18S64 and D18S51 on chromosome 18q21.2-21.3 in a large Northern Irish family ${ }^{3}, 4$. The gene encoding receptor activator of nuclear factor- $\kappa$ B (RANK: ref. 5), TNFRSF11A, maps to this region. RANK is essential in osteoclast formation ${ }^{6,7}$. We identified two heterozygous insertion mutations in exon 1 of TNFRSF11A in affected members of four families with FEO or familial Paget disease of bone (PDB). One was a duplication of $\mathbf{1 8}$ bases and the other a duplication of 27 bases, both of which affected the signal peptide region of the RANK molecule. Expression of recombinant forms of the mutant RANK proteins revealed perturbations in expression levels and lack of normal cleavage of the signal peptide. Both mutations caused an increase in RANK-mediated nuclear factor- $k$ $\mathrm{B}(\mathrm{NF}-\mathrm{KB})$ signalling in vitro, consistent with the presence of an activating mutation.

We studied the genetic basis of FEO in members of the large Northern Irish FEO family in which linkage to markers on $18 \mathrm{q}$ has been established. We also recruited a smaller American FEO family with affected individuals in four generations, and one affected male from a German family with three affected males in two generations who were diagnosed with osteolytic expansile PDB (ref. 8). Genetic linkage analysis in the American FEO fam-

$a$

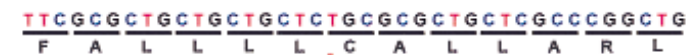

TTCGCGCTGCTGCTGCTCTGCGCGCTGCTCCTGCTCTGCGCGCTGCTCGCCCGGCTG

$b$

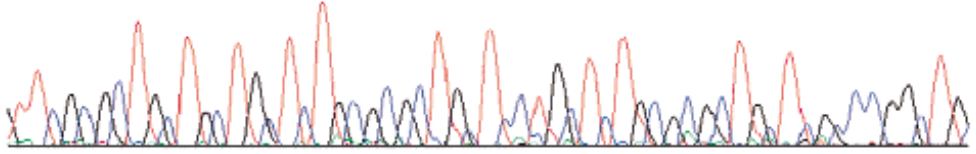

C

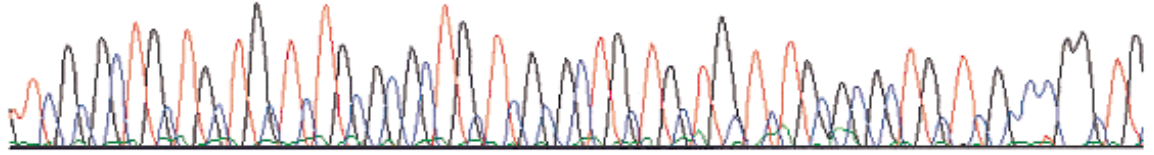

$d$

AGCAGCAGCAGCGC GAACAGCGGGCGGCGECGEGGGGCGC

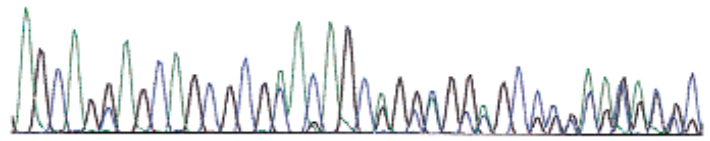

$e$

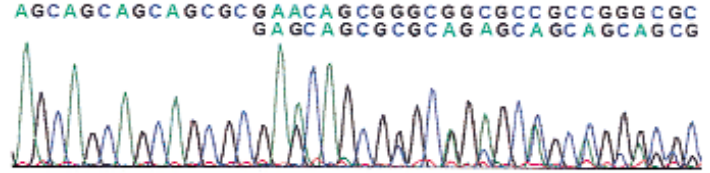

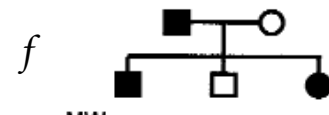

MW
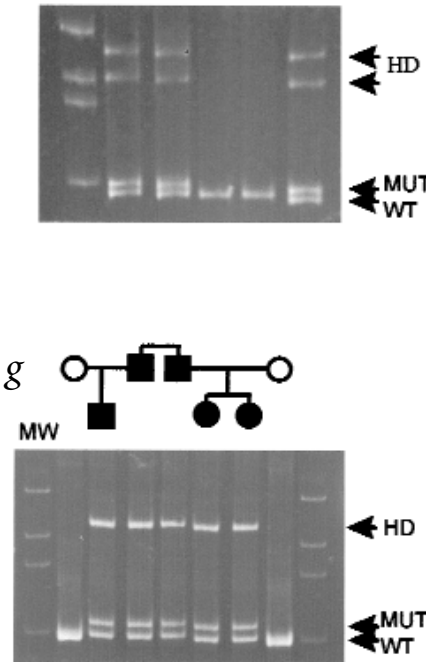

Fig. 1 Identification of TNFRSF11A mutations in FEO and familial PDB patients Sequence chromograms of the sense strand for normal control (a), FEO mutant (84dup18) (b) and PDB mutant (75dup27) (c) alleles are shown. The duplicated regions are underlined. Sequence chromograms of the antisense strand are shown to emphasize the mechanism of mutation, in heterozygous carriers of the FEO (d) and PDB (e) mutations. The sequence of the normal allele is shown above the sequence of the mutant allele. Confirmation of TNFRSF11A mutations by PAGE in part of the Northern Irish FEO pedigree shows the 18-bp duplication in affected individuals $(\boldsymbol{f})$ and in the PDB pedigree shows the 27-bp duplication in affected individuals (g). The positions of wild type (WT), mutant (MUT), heteroduplexes (HD) and molecular weight standards (MW) are indicated.

${ }^{1}$ Department of Medical Genetics, The Queen's University of Belfast, Belfast, UK. ${ }^{2}$ Department of Medicine and Therapeutics, University of Aberdeen, Aberdeen, UK. ${ }^{3}$ Department of Molecular Biology, Immunex Corporation, Seattle, Washington, USA. ${ }^{4}$ Department of Orthopaedic Surgery, Musgrave Park Hospital, Belfast, UK. ${ }^{5}$ Department of Medical Genetics, University of Antwerp, Antwerp, Belgium. ${ }^{6}$ Division of Bone and Mineral Diseases, Washington University School of Medicine, St. Louis, Missouri, USA. ${ }^{7}$ Second Department of Internal Medicine, Osaka City Hospital, Osaka, Japan. ${ }^{8}$ Orthopädische Universitatsklinik, Frankfurt/Main, Germany. Correspondence should be addressed to A.E.H. (e-mail: A.Hughes@qub.ac.uk). 


\begin{tabular}{ccc}
\hline \multicolumn{3}{c}{ Table 1 } \\
TNFRSF11A polymorphism IVS6+79A/G \\
\hline Genotype & Sporadic PDB & Controls \\
AA & 17 & 15 \\
AG & 29 & 33 \\
GG & 21 & 15 \\
Alleles & & \\
A & $63(0.47)$ & $63(0.50)$ \\
G & $71(0.53)$ & $63(0.50)$ \\
\hline
\end{tabular}

ily confirmed linkage to the FEO locus on $18 \mathrm{q}$ (data not shown), although their disease allele was transmitted on a different haplotype. Accordingly, the Northern Irish and American FEO families are unrelated. TNFRSF11A is a candidate for causing FEO because it maps to the $F E O / P D B 2$ region, is highly expressed in bone and has an important role in osteoclast differentiation and activity. The nucleotide sequence of TNFRSF11A mRNA was known at the start of our study. We characterized the intron-exon organization and the promoter region of TNFRSF11A by direct sequencing of genomic clones and PCR products and by vectorette PCR on PACs containing genomic DNA. A series of four GC boxes, which are recognized by the SP1 transcription factor, are present within the proximal 250 bases of the transcription start point. This region also contains several near-consensus GCbox sequences. There are no CAAT or TATA boxes. We identified several single-base polymorphisms in intron 6 . Analysis of their segregation within the Northern Irish FEO kindred supported localization of TNFRSF11A between markers D18S383 and D18S51. Analysis of clones in the Genebridge 4 radiation hybrid panel suggested a location in the region of D18S60 between WI9823 and SGC33768. TNFRSF11A is therefore located within the defined critical region for FEO.

We screened affected and unaffected members of the Northern Irish and American FEO families for mutations in all exons, intron-exon junctions, 5' and 3' UTRs, and the promoter region of TNFRSF11A by direct sequencing of PCR products. We identified an identical tandem duplication of bases 84-101 in exon 1 of all affected individuals tested (TNFRSF11A 84dup18; Fig. $1 a, b, d)$. This duplication segregated with the disease in both families. We confirmed the insertion in affected individuals by the presence of an allele of increased size, which was visible after polyacrylamide gel electrophoresis (PAGE; Fig. 1f). We did not detect insertions in unaffected members of the FEO families, 90 individuals with sporadic PDB or 158 controls (data not shown). We found an identical TNFRSF11A 84dup18 duplication in DNA from the only available affected member of the German family. We then performed mutation screening in members of four PDB families with evidence of possible linkage to 18q (ref. 9), which revealed a slightly larger duplication involving bases 75-101 (TNFRSF11A 75dup27; Fig. 1c,e) in exon 1 that co-segregated with the disease in one PDB family (Fig. $1 g$ ). We did not find TNFRSF11A mutations in the other three PDB families, or in cDNA prepared from affected bone of five patients with sporadic PDB. Both TNFRSF11A duplications share an identical 3' endpoint at base 101 and are likely to have arisen by reverse slippage during DNA replication.

We detected several TNFRSF11A polymorphisms that are not of functional significance. These include $-1 \mathrm{G} / \mathrm{A}$, close to the start position of transcription, and 30T/C, within the 5'-UTR, 9 bases before the initiation codon. We found an IVS1+5G/A polymorphism in the $5^{\prime}$ splice site of intron 1 . The rarer AG/gtaaa variant segregated with the Northern Irish FEO duplication, whereas we found the identical American mutation on a homozygous AG/gtaag background. This implies that these FEO mutations arose independently. The similarity of the phenotypes in both families suggests that splicing is unlikely to be affected by the IVS1+5G/A polymorphism. We identified a variant, V/A192, in exon 6, which encodes a predicted cysteine-rich pseudorepeat in the extracellular region of RANK. Valine (which shows homology at this position with mouse Rank) was more common than alanine at this site. V/A192 was in strong linkage disequilibrium with three intronic polymorphisms: IVS5$17 \mathrm{~T} / \mathrm{C}$, IVS6+79A/G and IVS6+166A/G. Intron 6 contained another cluster of polymorphisms at its $3^{\prime}$ end: IVS6-151A/G, IVS6-245A/G and IVS6-258A/C.

We typed DNA from 67 Northern-Irish patients with sporadic PDB and 63 age-matched controls for TNFRSF11A polymorphism IVS6+79A/G (Table 1). The distribution of genotypes in cases and controls did not deviate from Hardy-Weinberg equilibrium. We found no significant association between this polymorphism and sporadic PDB ( $\chi^{2}=0.13 ; P=0.72$ with 1 d.f. $)$, and no associations when polymorphisms in the promoter region of TNFRSF11A were typed in a smaller sample (data not shown). This shows that TNFRSF11A has, at most, a minor gene effect in the pathogenesis of sporadic PDB, a finding supported by other studies (A. Sparks et al., manuscript submitted).

We used mammalian expression plasmids encoding full-length wild-type TNFRSF11A (pWTRANK), FEO mutant RANK (pFEORANK) and PDB mutant RANK (pPDBRANK) in transient transfection and labelling experiments to assess the relative expression levels of the three different forms of RANK. Lower levels of recombinant receptor were expressed by pFEORANK ( $25 \%$ of wild type) and pPDBRANK (50\% of wild type) compared with pWTRANK transfectants (Fig. 2). In addition, the mutant RANK proteins expressed were larger than wild-type RANK (Fig. 2), and were of a size consistent with the lack of normal cleavage of the signal peptide. These results suggest that both mutations result in the inability of RANK protein to traffic normally to the cell surface.

We co-transfected plasmids pWTRANK, pFEORANK and pPDBRANK into mammalian cells with an NF- $\kappa B$-responsive luciferase reporter plasmid to assess whether the duplications in the RANK signal peptide found in FEO or familial PDB affect the functional capacity of the mutant receptor. Cell lysates contained up to a twofold higher level of luciferase activity when transfected

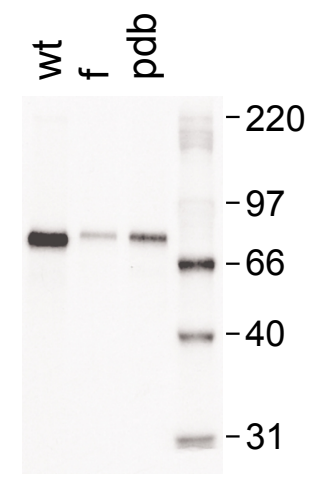

Fig. 2 Mutations result in decreased expression levels of RANK and lack of signal peptide cleavage. Metabolically labelled cell lysates from 293EBNA cells transfected with pWTRANK (w), pFEORANK ( $f$ ) and pPDBRANK (pdb) were immunoprecipitated with anti-RANK monoclonal antibody M331 and resolved by PAGE. The relative amounts of immunoprecipitated protein were the same using four different anti-human RANK monoclonal antibodies (data not shown). The larger apparent sizes of FEORANK and PDBRANK compared with wild-type RANK ( 3 kD larger) are consistent with lack of cleavage of the 30-aa (FEO) and 33-aa (PDB) signal peptides predicted by the GCG program SIGNALPEP. The sizes of protein molecular weight standards (in KD) are shown at right. 
$a$

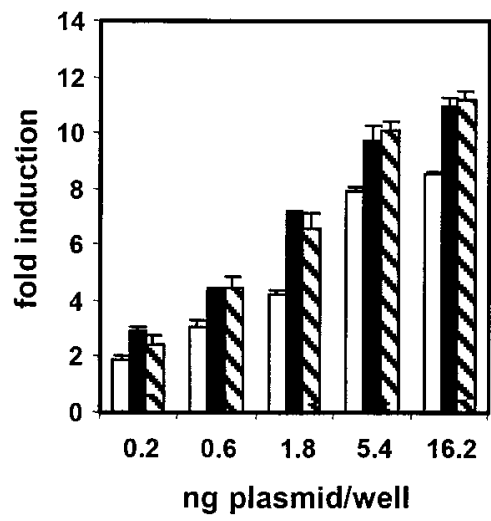

$b$

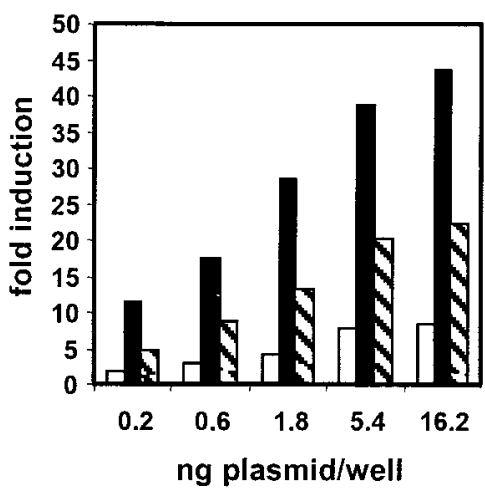

Fig. 3 The FEO and PDB mutations result in increased constitutive RANK signalling. a, RANK activation of an NF- $\mathrm{KB}$-responsive luciferase reporter was assessed in 293EBNA cells transfected with increasing amounts of pWTRANK, pFEORANK or pPDBRANK plasmid DNA. Two days post-transfection, cell lysates were collected and assayed for luciferase activity. Luciferase activity is presented as increase relative to control lysates from cells transfected with luciferase reporter plasmid alone. Values were normalized to levels of $\beta$-galactosidase. Reporter activity is shown from lysates from cells transfected with pWTRANK (open bars), pFEORANK (filled bars) and pPDBRANK (crosshatched bars). Data are means \pm standard deviation, $n=3$ from one representative experiment of three which gave comparable results. $\boldsymbol{b}$. The data shown in (a) adjusted for the relative expression levels of FEO RANK $(0.25 \times)$ and PDB RANK $(0.5 \times)$; wild type $=1 \times$, based on quantitation of the RANK protein bands shown in Fig. 2. Quantitation was performed on a STORM Phosphorimager using the ImageQuant program (data not shown). with pFEORANK or pPDBRANK compared with pWTRANK (Fig. $3 a$ ). These differences were amplified when the reporter activities were recalculated on the basis of the relative receptor expression levels (Fig. 3b). These results show that the FEO and PDB mutations result in increased constitutive activation of RANK.

FEO and familial PDB are similar disorders. They are distinguished clinically by the distribution of osteolytic lesions, with sparing of the axial skeleton in FEO. The Northern Irish and American FEO families share the same TNFRSF11A 84dup18 mutation and have very similar clinical features. Deafness is the earliest manifestation of the disease and often occurs before the age of ten. There is early loss of adult dentition due mainly to resorption in the cervical region of the teeth. Expansile osteolytic lesions present from the late teenage years until late middle age. Affected individuals usually develop one or two lesions, which are most prevalent in the tibia, ulna, humerus and femur. Amputation has been performed for symptom control in both families. No member of either family has had involvement of the skull or pelvis, which are common sites for PDB lesions. The small German family, which has the same $84 \mathrm{dup} 18$ mutation, appears to have more severe disease. The proband has large deformities affecting both hands, both tibiae and both femorae. His father died as a result of Paget sarcoma associated with a lesion in the femur and also had lesions affecting both arms and the skull, and his brother has similar, though less pronounced, skeletal deformities. The family with PDB that carries the TNFRSF11A 75 dup27 mutation is of Japanese origin. Most affected individuals presented in their teens and early twenties with bone pain or deformity. Unlike the FEO families, affected individuals have involvement of the axial skeleton with lesions in the spine, pelvis and mandible as well as at sites associated with lesions in FEO. All affected patients have dental problems and several have hearing impairment. These four families have similar activating mutations in TNFRSF11A. The inter- and intra-familial phenotypic variation may reflect subtle differences in the effects of the two mutations, differing genetic backgrounds or variation in expression of potential cytokine triggers of osteolysis.

The TNFRSF11A mutations that we have described produce short in-frame insertions within the signal peptide region of RANK. These insertions result in lower levels of recombinantly expressed RANK protein, a lack of normal cleavage of the RANK signal peptide and increased RANK-mediated activation of NF$\kappa \mathrm{B}$. The duplications occur in the ' $h$ ' or hydrophobic core region of the RANK signal peptide ${ }^{10}$. The length of this region is critical for proper signal peptidase cleavage ${ }^{11}$. A possible mechanism to explain our findings is that lack of proper RANK signal peptide cleavage results in higher intracellular accumulation of defective
RANK translation products in compartments of the secretion pathway. This accumulation then leads to a higher incidence of receptor self-association, and thus, increased constitutive RANK signal transduction. In addition to RANK, several other members of the TNF-receptor superfamily transmit signals using the same downstream pathways. The fine-tuning mechanisms for regulating their wide-ranging roles in cellular proliferation, differentiation, survival and apoptosis remain to be elucidated. All of these may be critical for maintaining normal bone metabolism. Our results highlight the importance of regulatory factors in the RANK-mediated osteoclastogenesis pathway in diseases of bone metabolism.

\section{Methods}

Genomic organization of TNFRSF11A. We identified the position of each intron within TNFRSF11A and obtained end sequence using several methods, including DNA sequencing of PCR products generated with primer pairs designed at intervals within the CDNA and vectorette PCR on genomic clones. We selected PACs 108M4 and 131H23 from the RPCI1 PAC library $^{12}$ by hybridization of TNFRSF11A cDNA to high-density gridded filters (supplied by the UK HGMP Resource Centre). We made vectorette libraries of pooled DNA from both PACs using AluI, RsaI, StuI, NruI, SmaI and HaeIII (ref. 13).

Genomic DNA amplification. We amplified exon 1 of TNFRSF11A from genomic DNA using forward primer 5'-TGGGGTGCGGCAAGG CAGGAG-3' and reverse primer 5'-AAGGCGGAGGAGCCAGGATGC-3'. We performed PCR in reactions $(20 \mu \mathrm{l})$ using Taq DNA polymerase $(0.5 \mathrm{U}$; Qiagen), $1 \times$ buffer and solution Q, dNTPs $(200 \mu \mathrm{M})$, primers $(0.3 \mu \mathrm{M})$ and DNA (50 ng). PCR conditions were as follows: initial denaturation at $97^{\circ} \mathrm{C}$ for $3 \mathrm{~min}$ followed by 35 cycles of $97^{\circ} \mathrm{C}$ for $1 \mathrm{~min}$; annealing at $65^{\circ} \mathrm{C}$ in the first 10 cycles, then $63{ }^{\circ} \mathrm{C}$ for $1 \mathrm{~min}$ and extension at $72{ }^{\circ} \mathrm{C}$ for $1 \mathrm{~min}$. We amplified polymorphism IVS6+79A/G by amplification of IVS6+14 to IVS6+300 using forward primer 5'-GAGCCTGTTGGTTGATAGACGTG-3 and FAM-labelled reverse primer $5^{\prime}$-ACTTGTGACCACCATCATCCCAC $-3^{\prime}$ as described above (annealing at $60^{\circ} \mathrm{C} / 55^{\circ} \mathrm{C}$ ). This polymorphism was typed using the BESS T-Scan system (Epicentre). Primers and conditions for amplification of all other exons are available on request.

Mutation detection and analysis. We purified PCR products on Wizard columns (Promega) and sequenced with an ABI377 instrument using BigDye terminator cycle sequencing chemistry. We included 5\% DMSO in sequencing reactions for exon 1 . Mutations were confirmed by size variation after electrophoresis on 5\% polyacrylamide gels run in $1 \times \mathrm{TBE}$ buffer and stained with ethidium bromide. We excised individual alleles and reamplified by PCR before sequencing as above.

Wild-type and mutant TNFRSF11A expression vector construction. We used two sets of synthetic oligonucleotide linker pairs to recreate the $5^{\prime}$ coding region of wild-type human TNFRSF11A (WTRANK) and to create the corresponding regions of FEO TNFRSF11A (FEORANK) and PDB 
TNFRSF11A (PDBRANK). For WTRANK, we synthesized four oligonucleotides: 1, 5'-TCGACGCCATGGCCCCGCGCGCCCGGCGGCGCCGC CCGCTGTTCG-3'; 2, 5'-CGCTGCTGCTGCTCTGCGCGCTGCTCGCCCGGCTGCA-3; 3, 5'-GCCGGGCGAGCAGCGCGCAGAGCAGCAGCA GCGCGAACAGC-3'; 4, 5'-GGGCGGCGCCGCCGGGCGCGCGGGGCC ATGGCG-3'. These oligonucleotide sets encode the 5' coding region of TNFRSF11A from the initiator codon through the PstI site (nt 39-112). For FEORANK, we replaced oligonucleotides 2 and 3 with 5'-CGCTGCTGCTG CTCTGCGCGCTGCTCCTGCTCTGCGCGCTGCTCGCCCGGCTGCA-3 (5) and 5'-GCCGGGCGAGCAGCGCGCAGAGCAGGAGCAGCGCGCAG AGCAGCAGCAGCGCGAACAGC-3' (6). For PDBRANK, we replaced oligonucleotides 2 and 3 with 5'-CGCTGCTGCTGCTCTGCGCGCTGCT CGCGCTGCTGCTG-3'(7), 5'-GCGAGCAGCGCGCAGAGCAGCAGCA GCGCGAACAGC-3' (8), 5'-CTCTGCGCGCTGCTCGCCCGGCTGCA-3' (9) and 5'-GCCGGGCGAGCAGCGCGCAGAGCAGCAGCAGC-3' (10). Oligonucleotide pairs were phosphorylated with ATP and T4 polynucleotide kinase and allowed to anneal following heat inactivation of the kinase. We ligated oligonucleotide pairs $1 / 4$ and $2 / 3$ to the downstream coding fragment of TNFRSF11A (PstI-NotI fragment from pcdna3:huRANK; ref. 5) and a mammalian expression vector backbone to generate pWTRANK. Alternatively, oligonucleotide pairs $1 / 4$ and 5/6 were ligated to downstream TNFRSF11A and vector DNA fragments to generate pFEORANK, and oligonucleotide pairs $1 / 4,7 / 8$ and $9 / 10$ were ligated to downstream TNFRSF11A and vector DNA fragments to generate pPDBRANK. We verified all constructs by dye termination sequencing of both DNA strands.

Cell transfection and reporter assay. An NF- $\mathrm{KB}-$ responsive reporter plasmid using a promoter with three NF- $\mathrm{KB}$-binding sites fused to a luciferase reporter has been described ${ }^{14}$. We transfected the reporter plasmid $(40$ $\mathrm{ng} /$ well) alone or in combination with varying amounts of pWTRANK, pFEORANK or pPDBRANK plasmids into 293EBNA cells (Invitrogen) using the DEAE-dextran transfection method. A plasmid expressing $\beta$ galactosidase $(25 \mathrm{ng} /$ well $)$ was included as an internal transfection efficiency control. Total amounts of plasmid DNA for each transfection were equal-

1. Osterberg, P.H. An unusual familial disorder of bone: response to calcitonin in Bone Disease and Calcitonin (ed. Kanis, J.A.) 181-185 (Armour Pharmaceutical Company, London, 1976).

2. Osterberg, P.H. et al. Familial expansile osteolysis. A new dysplasia. J. Bone Joint Surg. Br. 70, 255-260 (1988).

3. Hughes, A.E. et al. Genetic linkage of familial expansile osteolysis to chromosome 18q. Hum. Mol. Genet. 3, 359-361 (1994).

4. Hughes, A.E. \& Barr, J. Familial expansile osteolysis: a genetic model of Paget's disease. in The Molecular Biology of Paget's Disease (ed. Sharpe, P.T.) 179-199 (RG Landes, Heidelberg, 1996).

5. Anderson, D.M. et al. A homologue of the TNF receptor and its ligand enhance T-cell growth and dendritic-cell function. Nature 390, 175-179 (1997).

6. Nakagawa, N. et al. RANK is the essential signaling receptor for osteoclast differentiation factor in osteoclastogenesis. Biochem. Biophys. Res. Commun. 253, 395-400 (1998).

7. Hsu, H. et al. Tumor necrosis factor receptor family member RANK mediates osteoclast differentiation and activation induced by osteoprotegerin ligand. Proc. Natl Acad. Sci. USA 96, 3540-3545 (1999).

8. Enderle, A. \& von Gumppenberg, S. [Osteitis deformans (Paget)—or a tarda- ized by the addition of empty vector. We collected cell lysates from transfected cultures after $48 \mathrm{~h}$ incubation using reporter lysis buffer (Promega). We measured luciferase activity in cell lysates using luciferase substrate (Promega) and an EG\&G/Berthold Luminometer. Relative luciferase activity was normalized to the $\beta$-galactosidase activity in each sample.

Metabolic labelling. We transfected subconfluent 293EBNA cells in 6-well plates with pWTRANK, pFEORANK or pPDBRANK ( $1 \mu \mathrm{g} /$ well $)$. Cells were metabolically labelled $48 \mathrm{~h}$ following transfection for $15 \mathrm{~min}$ with $\left[{ }^{35} \mathrm{~S}\right]$-cysteine/methionine $(50 \mu \mathrm{Ci} / \mathrm{ml}$; Promix, Amersham) in cysteinefree, methionine-free DMEM (Gibco). Cells were washed twice with PBS, collected with PBS/0.1\% Triton X-100 and clarified cell lysates immunoprecipitated with anti-human RANK antibody M331 (ref. 5; $10 \mu \mathrm{g} / \mathrm{ml}$ ) and protein A- and G-Sepharose (Pharmacia). Immune complexes were resolved by PAGE and radiolabelled proteins detected by autoradiography.

GenBank accession numbers. Human TNFRSF11A mRNA, AF018253 mouse Tnfrsf11a, mRNA AF019046.

\section{Acknowledgements}

We thank the clinicians and families for participation; C. Huang, M. Timour, J. Bertles, D. McGibbon and G. Taylor for technical support; N. Nevin, G. Means, J. Derry and D. Cosman for helpful discussions; and the UK Human Genome Mapping Project Resource Centre. S.H.R. was supported by grants from the Wellcome Trust, Arthritis Research Council (UK), the National Association for Relief of Paget's Disease (UK) and the Paget Foundation (USA).

Received 15 August; accepted 15 November 1999 type of a hereditary hyperphosphatasia.] Arch. Orthop. Trauma Surg. 94
127-134 (1979).

9. Haslam, S.l. et al. Paget's disease of bone: evidence for a susceptibility locus on chromosome 18q and for genetic heterogeneity. J. Bone Miner. Res. 13 911-917 (1998).

10. von Heijne, G. Signal sequences. The limits of variation. J. Mol. Biol. 184 99-105 (1984)

11. Nilsson, I., Whitley, P. \& von Heijne, G. The $\mathrm{COOH}$-terminal ends of internal signal and signal-anchor sequences are positioned differently in the ER translocase. J. Cell Biol. 126, 1127-1132 (1994).

12. Ioannou, P.A. \& de Jong, P.J. Construction of bacterial artificial chromosome libraries using the modified P1 (PAC) system. in Current Protocols in Human Genetics (ed. Dracopoli, N.C. et al.) 5.15.1-5.15.24 (John Wiley and Sons, New York, 1996).

13. Ogilvie, D.J. \& James, L.A. End rescue from YACs using the vectorette. in Methods in Molecular Biology Vol. 54 (ed. Markie, D.) 131-138 (Humana Press, Totowa, New Jersey, 1995).

14. Mitchell, T. \& Sugden, B. Stimulation of NF-K B-mediated transcription by mutant derivatives of the latent membrane protein of Epstein-Barr virus. $J$. Virol. 69, 2968-2976 (1995). 